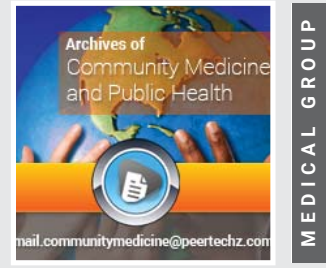

\section{Health Alert Network}

\author{
Dheeraj Sharma ${ }^{1 *}$ and Ansuman Panigrahi ${ }^{2}$ \\ ${ }^{1}$ Department of Community Medicine, Muzaffarnagar Medical College, Muzaffarnagar, Uttar Pradesh, \\ India \\ 2Department of Community Medicine, Kalinga Institute of Medical Sciences, KIIT University, \\ Bhubaneswar, Odisha, India
}

Received: 28 December, 2019

Accepted: 18 February, 2020

Published: 19 February, 2020

*Corresponding author: Dr. Dheeraj Sharma, Assistant Professor, Department of Community Medicine, Muzaffarnagar Medical College, Muzaffarnagar-251001, Uttar Pradesh, India, Tel: +918249143067; E-mail: sharma.dheeraj10@gmail.com ORCiD: https://orcid.org/0000-0002-4046-5035 https://www.peertechz.com

\section{Check for updates}

"Always plan ahead. It wasn't raining when Noah built the Ark." Having said that quote from Richard Cardinal Cushing about emergency preparation, let us move on to our topic "Health Alert Network".

The Centers for Disease Control and Prevention (CDC) is the leading national public health institute in the United States. The CDC uses Health Alert Network (HAN) for sharing information about urgent public health incidents with public information officers, public health practitioners, clinicians, and public health laboratories.

The HAN is a strong national program as it provides vital health information and the infrastructure at different levels; such as federal, state, territorial, tribal, local, and beyond. Categories of HAN messages are into Health Alert, Advisory, Update, and Info Service based on a decreasing level of importance warranting attention by health personnel.

The CDC claims transmission of HAN messages to over one million recipients and coverage of over $90 \%$ of their populations under HAN programs. However, the jurisdictional distribution of these one million recipients and the basis of $90 \%$ coverage of populations under HAN is not present on the CDC portal [1].

There are some practical roadblocks in the proper functioning of HAN. Only delivering the messages to HAN stakeholders does not solve the problem. Apart from all stakeholders of CDC's HAN, the public and the patient should also receive HAN messages. Everyone in this chain should understand the message, know its importance in their context and their role in such situations.

CDC's HAN and its stakeholders are using one-way (e.g., Media) as well as two-way (e.g., Interpersonal) communication, but the effectiveness of one-way communication is always lesser than two-way as a matter of fact. It is also difficult to ensure the receipt of one-way communication.
Even if the CDC is effectively passing one-way communication HAN to its stakeholders and stakeholders are passing it to the public and patient, all those who are not in touch may miss it or receive it quite late. There is a possibility of inoperable HAN occasionally, which halts the whole system until one corrects it [2].

The CDC sends HAN messages to its stakeholders at the time of emergency, and stakeholders then deliver it to the public and patient. This process practically takes some time and this time lag in receiving critical information by end-users can be catastrophic in the emergency.

Many of the HAN messages have dealt with infectious diseases [3]. People with symptomatic disease mostly go to clinicians while people with asymptomatic disease, less symptomatic disease, and healthy people fall under the scope of public information officers and public health practitioners. People are mostly unaware of how asymptomatic, less symptomatic, and healthy people play their role unknowingly in disease transmission and propagation.

Some of the HAN messages have been on natural disasters [4]. The public needs to know their role in such a situation beforehand so that they can cope up with the situation in case of an emergency until the response team reaches on the site.

Let us summarize the problem. CDC's HAN consist of two sub-segment top to bottom. The top segment is HAN messages from the CDC to its stakeholders and the bottom segment is the delivery of these messages from stakeholders to end-users. The top segment is quicker, robust and interactive by design in comparison to the bottom segment; which is a bit slow, passive and not that much end-user friendly.

The design of HAN makes health care providers part stronger than the public part, who should be the actual beneficiary. The program deals more with the role and responsibility of 
healthcare workers than the public. There is a possibility of time lag in receipt of messages by intended recipients due to one-way communication used in many places. Inoperable HAN causes the collapse of this whole functionary sometimes.

The timely interpersonal communication between CDC, its stakeholders, and the public is the priority of the moment. The objective of this opinion piece is to make the HAN more interactive and quickly responding along with the involvement of the CDC, its stakeholders, and the public. There is a requirement of extra resources for involving the public in this chain of the message and for making communication timely and interactive. Charitable contributions and philanthropic grants can become the source of funding for it.

We have some recommendations for the betterment of this program. The CDC should upload the jurisdictional distribution of one million stakeholders of HAN messages on its portal. It will not only help people to identify CDC's HAN service providers in their area but will also be enlightening to researchers and policymakers.

Objective and subjective assessment of CDC's stakeholders, the public and the patient is necessary to know and ensure their understanding of HAN messages. The email or telephone will save time, but face to face communication will consume more resources and make the process lengthy. This will unveil the operational difficulties in the whole process and percentage coverage of the population under HAN can be better expressed after this.

Both, two-way and one-way communication should go on simultaneously. Contact details (e.g., Phone number, email id, address) of the public and stakeholders of respective areas should be available to each other for emergency contact purposes. Stakeholders should pass the relevant element of HAN message to the public of their area at the earliest possible to minimize any loss of public and economy in an emergency. These messages should reach the public in simple language explaining their role in such a situation. The proactive involvement of the public and a clear understanding of the role beforehand is crucial.

If HAN becomes inoperable, HAN duty officers should perform system and other tests quickly along with the help of experts to maintain and restore the proper operation.

When the clinicians are busy dealing with cases of the disease, public information officers and public health practitioners must focus on the rest of the public. They should explain to the public about any possibility of latent disease in the community that is not fully manifest but still transmitting. The public should be aware of their role in preventing disease transmission and during natural disasters.

The recommended plan needs implementation, monitoring, evaluation, and reassessment to know the attainment of its intended objective. The above recommendation implies that it will not only make the program better by clearing the obstacles in its way but will also be more helpful to the public due to its more timely and interactive nature. As a result, the overall damage will reduce.

The drawback of this recommended plan is the requirement of an ample amount of resources (health manpower, money, material and time). The deficit in health manpower and time appears to create hindrance in its implementation.

Diagnosing the diseases in the subclinical stage, scientific prediction of natural disasters and the use of artificial intelligence in the program can play a big role in future researches.

At last, these lines by Benjamin Franklin appear to fit in the situation...... Tell me and I forget. Teach me and I remember. Involve me and I learn.

\section{References}

1. Centers for Disease Control and Prevention. Health Alert Network (HAN) (2020) Resour Emerg Heal Prof 2016. Link: http://bit.ly/39HL9m8

2. Response E, State L, Building O, Moines D (2013) HAN Resource Manual. lowa Dep Public Heal 1-8. Link: http://bit.ly/2vF26iu

3. Centers for Disease Control and Prevention (2020) HAN Messages. Emerg Prep Response 2020. Link: http://bit.ly/39MJP1w

4. Centers for Disease Control and Prevention (2017) HAN Messages. Emerg Prep Response 2017. Link: http://bit.ly/37BQ0nl

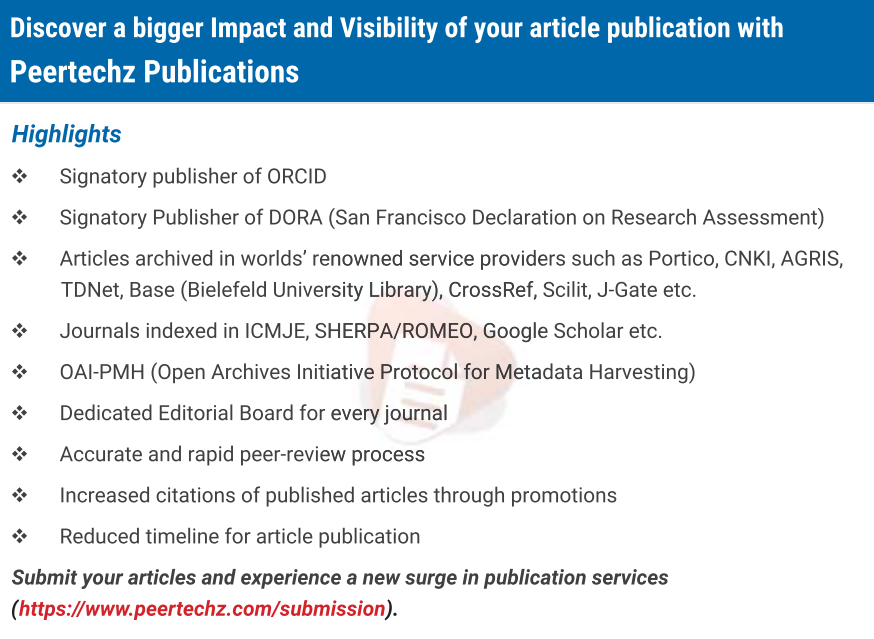
Peertechz journals wishes everlasting success in your every endeavours.

Copyright: @ 2020 Sharma D, et al. This is an open-access article distributed under the terms of the Creative Commons Attribution License, which permits unrestricted use distribution, and reproduction in any medium, provided the original author and source are credited. 